\title{
eJRIEPS
}

Ejournal de la recherche sur l'intervention en éducation physique et sport

$18 \mid 2009$

Varia

\section{Construire une activité collective de travail dans une classe d'EPS en 'Réseau ambition réussite' : entre masquage et ostentation}

Olivier Vors et Nathalie Gal-Petitfaux

\section{(2) OpenEdition}

1 Journals

Édition électronique

URL : http://journals.openedition.org/ejrieps/5518

DOI : $10.4000 /$ ejrieps.5518

ISSN : 2105-0821

Éditeur

ELLIADD

Référence électronique

Olivier Vors et Nathalie Gal-Petitfaux, "Construire une activité collective de travail dans une classe d'EPS en 'Réseau ambition réussite' : entre masquage et ostentation », eJRIEPS [En ligne], 18 | 2009, mis en ligne le 01 juillet 2009, consulté le 18 mars 2021. URL : http://journals.openedition.org/ejrieps/ 5518 ; DOI : https://doi.org/10.4000/ejrieps.5518

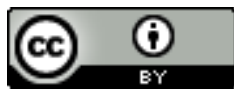

La revue eJRIEPS est mise à disposition selon les termes de la Creative Commons Attribution 4.0 International License. 


\section{Construire une activité collective de travail dans une classe d'EPS en 'Réseau}

\section{ambition réussite' : entre masquage et ostentation}

Olivier Vors \& Nathalie Gal-Petitfaux

Université Blaise Pascal, Clermont-Ferrand, France.

Résumé

Dans le contexte difficile des RAR, l'activité des élèves s'oppose souvent à celle de l'enseignant, ce qui rend l'activité collective de travail dans la classe particulièrement difficile à construire et instable. Seuls les enseignants expérimentés parviennent à installer et maintenir une activité collective viable propice à l'apprentissage. Cette recherche vise à comprendre comment une telle activité collective de travail peut s'installer en classe et perdurer au cours de la leçon.

L'étude est conduite en référence au paradigme de l'action située, et à la théorie du cours d'action (Theureau, 2009) appréhendant l'activité collective à partir des activités individuellessociales. Elle est menée dans un établissement classé RAR, avec une classe de $5^{\text {ème }}$, lors d'un cycle de gymnastique par ateliers. Six élèves et leur enseignant expérimenté ont été filmés et ont suivi des entretiens d'autoconfrontation.

Les résultats montrent que les activités contradictoires des élèves et de l'enseignant se coordonnent pour produire une activité collective viable compatible avec les exigences de travail scolaire de l'enseignant. Cette coordination repose sur un processus d'ostentation/masquage des actions de chacun.

\section{Introduction}

Activités contrariées dans une classe 'Réseau ambition réussite'

Notre étude s'intéresse à la façon dont une activité collective de travail peut s'installer en classe et perdurer au cours de la leçon, avec des élèves peu enclins à s'engager dans un travail scolaire. Son objectif est de comprendre comment les activités individuelles contradictoires de l'enseignant et des élèves peuvent s'articuler pour atteindre un seuil de viabilité du travail collectif acceptable pour tous. Le contexte de l'étude est celui de l'enseignement scolaire de la gymnastique en Éducation physique et sportive (EPS), dans une classe difficile d'un Réseau ambition réussite (RAR), avec des enseignants réussissant 
(Chauveau, 2001). Pour l'auteur, les maîtres réussissant en ZEP sont ceux qui parviennent le mieux à mettre les élèves en activité apprenante pendant la plus grande partie du temps scolaire. Ils ont entre autres la capacité à gérer le groupe-classe, à y organiser la vie scolaire (le «vivre ensemble») et l'activité cognitive collective («l'apprendre ensemble»).

L'apparition des RAR correspond au troisième plan de relance de l'éducation prioritaire de 2005. Depuis 2009, 254 réseaux ont été listés, correspondant aux collèges et à leurs écoles primaires les plus en difficulté de France. Dans ces contextes, la mise au travail du collectif d'élèves est un véritable défi pour les enseignants dont l'activité d'instruction est constamment contrariée, empêchée, interrompue par des pratiques transgressives chez les élèves (Millet \& Thin, 2005). En classe, ces derniers ont une forte tendance à décrocher des tâches scolaires prescrites (Guérin, Pasco \& Riff, 2008), à refuser d'apprendre, à s'opposer à l'autorité scolaire pouvant conduire à la déscolarisation (Glasman, 2003). Et leur sociabilité juvénile entre souvent en dissonance avec la socialisation visée par l'école (Vors \& GalPetitfaux, 2008a). Cette situation place ces adolescents sous une triple contrainte scolaire/parentale/sociale (Lahire, 2004). Ce profil dissonant va même jusqu'à une situation de concurrence. De leur côté, les enseignants vivent ces situations scolaires comme particulièrement instables et épuisantes par la nécessité de constamment faire de la discipline et de remettre les élèves au travail (e.g. Monfroy, 2002). Seuls les enseignants réussissant (Chauveau, 2001) parviennent à installer et maintenir, dans la classe, une activité collective de travail (Vors \& Gal-Petitfaux, 2008b).

D'autres études relatives à l'enseignement en milieu difficile s'intéressent particulièrement aux relations entre l'activité de l'enseignant et celle des élèves (e.g. Kherroubi \& Rochex, 2004). Les analyses convergent vers l'idée que l'instauration d'une activité collective de travail durable dans la classe est une épreuve laborieuse pour l'enseignant, occasionnant une importante dépense d'énergie et une fatigue, provoquées par les problèmes de discipline: « faire tenir un certain nombre d'élèves le temps voulu dans un lieu précis et dans des conditions de communication à peu près acceptables n'est plus alors le point de départ de la situation scolaire mais un de ses objectifs » (ibid., p. 172). Face aux contrariétés qui peuvent se présenter dans une classe RAR, l'installation d'une activité collective de travail chez les élèves ne va pas de soi. Dans les deux paragraphes suivants, nous présentons comment différents champs de recherche traitent de la coordination des activités individuelles et de l'activité collective. 
Activités individuelles et activité collective

À l'heure actuelle, différents champs de recherche orientent leurs études sur le collectif (Saury, 2008, pour une revue), appréhendant la construction de l'activité collective selon divers éclairages. Theureau (2006) distingue trois grandes approches méthodologiques : l'individualisme, le collectivisme et le situationnisme. Premièrement, l'individualisme méthodologique se fonde sur une conception cognitiviste de l'activité comme système de traitement de l'information (Newell \& Simon, 1972, pour ne citer qu'un ouvrage fondateur). L'activité individuelle est appréhendée comme le produit de la mise en œuvre de représentations individuelles dans un monde prédéfini. La focale est orientée vers l'individu, l'activité collective étant considérée comme le fruit de représentations individuelles partagées. Deuxièmement, le collectivisme méthodologique prend le contre-pied de cette vision en accordant la primeur à l'activité collective. Cette approche permet d'avoir une vision d'ensemble sur l'autonomie relative des collectifs munis de leur interface. II s'agit notamment de l'ethnométhodologie ou plus généralement de l'interactionnisme, de l'analyse conversationnelle, ou encore du courant de la cognition sociale distribuée (Hutchins, 1995). Enfin, la troisième conception est le "situationnisme méthodologique » (Theureau, 2006, p. 90). Il correspond à une voie moyenne entre l'individualisme et le collectivisme : le collectif est approché à la fois comme activité collective et comme intégrant l'activité individuelle. Plus précisément, l'activité collective est envisagée comme une articulation d'activités individuelles de plusieurs acteurs munis de leurs interfaces. "Les individus interagissent entre eux, non pas directement, mais d'une façon toujours médiée par leurs "interfaces situationnelles" propres (chacun interagissant avec sa propre situation) " (Saury, 2008, 45). Les recherches concernées appartiennent au champ de l'anthropologie cognitive nord-américaine (Dougherty, Lave, Suchman, etc...), de l'ergonomie cognitive (Theureau, 2006) et même dans certaines approches du courant de l'écologie (Hastie \& Siedentop, 2006, pour une revue).

C'est dans cette troisième approche méthodologique que se situe notre étude. Ainsi nous définissons l'activité collective comme " un espace de travail partagé où les interactions sont à la fois directes (communications verbales en continu et visibilité des comportements) et médiées, de manière simultanée ou décalée dans le temps, par un système informatique et par d'autres artéfacts (par exemple, document papier, synoptique) " (Filippi, 1994, p. 40). Cette définition minimale ne préjuge pas a priori de la nature de l'activité collective. Dans le cadre du situationnisme méthodologique l'activité collective a été étudiée au travers différents 
objets de recherche 1/ l'activité coopérative : sa manifesteté mutuelle (Sperber \& Wilson, 1986), ses savoirs mutuels (Leplat, 1994), son contexte partagé (Salembier \& Zouinar, 2004) et 2/ la forme d'organisation de l'activité collective (Baluteau, 2003 ; Durand, Saury \& Sève 2006 ; Hastie \& Siedentop, 2006). Pour étudier l'activité collective de la classe, nous nous intéresserons plus particulièrement à ce deuxième objet.

\section{Les formes d'interaction révélatrice de l'activité collective}

La vie collective dans la classe a été d'abord une préoccupation des recherches appartenant au courant écologique de la classe initié par Doyle en 1977. Ce champ de recherche s'intéresse aux interactions entre le monde vivant et son milieu naturel. La classe est appréhendée comme un écosystème vivant, c'est-à-dire un système formé par l'ensemble des individus (enseignant et élèves) et de l'environnement (situations pédagogiques et ressources matérielles disponibles), en interdépendance dynamique à la recherche d'un équilibre (Hastie \& Siedentop, 2006).

La dynamique de la classe a été aussi étudiée par le courant de l'ethnographie de l'école. Cette approche c'est particulièrement intéressée aux conditions d'instauration d'une activité collective de travail dans la classe (Coulon, 1988, pour une revue). Les observations relevées montrent qu'il est essentiel de parvenir à instaurer et à stabiliser des formats d'interactions dans les classes pour pouvoir structurer une activité collective de travail viable chez les élèves. Ces recherches étudient les organisations scolaires en tant que configuration (Baluteau, 2003), c'est-à-dire un ensemble de relations d'interdépendance entre les individus qui prend une forme stable et qui émerge de leurs relations. La cohérence d'une activité collective tient au fait que les acteurs se trouvent dans des formes typiques d'interaction, avec les personnes et les objets présents dans la situation, qui coordonnent et cadrent leurs actions. Cette notion de format d'interaction rejoint celle de « cadre de l'expérience » avancée par Goffman (1991) et de "cadre » repris par Joseph (1998), pour signifier que toute expérience, toute activité sociale, est cadrée. Un cadre est un dispositif cognitif et pratique d'organisation de l'expérience sociale qui nous permet de comprendre ce qui nous arrive et d'y prendre part. Un cadre structure aussi bien la manière dont nous définissons et interprétons une situation que la façon dont nous nous engageons dans un cours d'actions. II est en somme une forme typique d'organisation des interactions sociales et de l'expérience collective dans une situation donnée (Gal-Petitfaux \& Vors, sous presse).

S'intéresser à l'activité collective revient alors à repérer la structure qui relie les individus, c'est-à-dire repérer le processus d'élaboration de formes d'interactions sociales structurant 
l'activité collective en classe. Par exemple, Marchive (2003) montre le rôle important des formes d'organisation de l'activité collective dans une classe de Zone d'Éducation Prioritaire (ZEP) sur les conditions de diffusion des savoirs, et, plus largement, dans l'instauration de l'ordre dans la classe. Amigues et Zerbato-Poudou (2000) vont plus loin en montrant que l'instauration de "formats d'interactions " stables dans une classe en milieu difficile permet d'instaurer un code commun de significations entre les élèves, participe à coordonner les actions individuelles des élèves avec celles de l'enseignant et favorise l'apprentissage. La mise en évidence de formats d'interactions comme condition d'instauration d'une activité collective viable dans la classe concerne l'étude de différentes situations d'enseignements scolaires comme : l'écriture (Amigues \& Zerbato-Poudou, 2000), les mathématiques (Mottier Lopez, 2006), la lecture (Veyrunes, Gal-Petitfaux \& Durand, 2007), l'EPS (d'ArripeLongueville, Fleurance \& Winnykamen, 1995 ; Saury, 2008).

\section{Cadre d'étude}

Cette recherche s'intéresse à la vie dans la classe, selon une approche descriptive et compréhensive orientée "activité " (Barbier \& Durand, 2003). Elle vise à comprendre comment une activité collective de travail peut s'installer dans une classe d'un collège en RAR et perdurer au cours de la leçon. En d'autres termes, comment les activités individuelles du professeur et de ses élèves, propices à se contrarier, peuvent s'articuler pour produire un engagement relativement durable du collectif d'élèves au travail? Quelles sont plus particulièrement les formes d'articulation de ces activités individuelles lors de leçons de gymnastique organisées selon un dispositif par ateliers?

Le cadre théorique utilisé pour ces études de cas est celui de l'action située (Lave \& Wenger, 1991) en référence au modèle sémiologique du cours d'action et selon un situationnisme méthodologique (Theureau 2006, 2009). Suivant ce cadre, l'activité collective est envisagée comme une articulation d'activités individuelles de plusieurs acteurs munis de leurs interfaces. Le collectif n'est pas considéré comme une "totalité constituée", mais comme une "totalité" constamment "détotalisée" par l'activité de ses composants, collectifs plus petits et individus, et il doit être abordé à partir de l'activité des pratiques individuelles (Sartre, 1960, p. 432).

Quatre présupposés théoriques éclairent l'objet d'étude de l'activité collective de travail. Premièrement l'activité de l'acteur est considérée comme un accomplissement pratique situé, c'est-à-dire indissociable de la situation dans laquelle elle prend forme et se manifestant par 
des comportements observables. Deuxièmement, l'activité humaine est vécue, au sens où elle donne lieu à une expérience subjective pour l'acteur. Selon le modèle du cours d'action, l'expérience est un niveau d'organisation relativement autonome par rapport à d'autres niveaux possibles d'analyse de l'activité et il peut donner lieu à des descriptions et explications valides (Theureau, 2009). Pour rendre compte de l'expérience, il est nécessaire d'atteindre les composantes des significations construites par les acteurs au cours de leur activité: leurs intentions, leurs interprétations, leurs perceptions et leurs émotions. Troisièmement, l'activité individuelle est sociale en tant qu'elle s'inscrit constamment dans un cadre collectif et où autrui appartient à la conscience préréflexive de l'acteur (ibid.). Autrement dit, dans l'activité de l'acteur, son environnement social fait partie de son expérience personnelle. Autrui participe donc fondamentalement à l'activité de chaque acteur, même lorsque celui-ci est étudié dans une situation dite individuelle, voire solitaire. Nous accédons à cette activité individuelle-sociale (Theureau, 2006) en recourant à la signification que les acteurs attribuent à leurs interactions avec autrui à l'aide d'entretien d'autoconfrontation. Quatrièmement, l'activité collective provient de l'articulation des activités individuelles-sociales.

L'étude de cas relatée s'est faite dans un collège RAR de la banlieue lilloise. Elle concerne l'enseignement de l'EPS en gymnastique par ateliers durant un cycle de huit séances, avec une classe de $5^{\mathrm{e}}$ de 18 élèves jugés très difficiles par l'équipe pédagogique. Les sujets de l'étude sont un enseignant réussissant (EG W) ainsi que six de ses élèves volontaires. Les élèves de la classe devaient acquérir des éléments gymniques pour ensuite les lier et présenter un enchaînement en fin de cycle. Chaque séance se déroulait dans un grand gymnase. Elle débutait par une phase d'installation du matériel (les agrès gymniques composant chaque atelier) et était suivie par une phase de travail gymnique proprement dite. La classe était divisée en quatre ateliers de travail se décrivant ainsi : un atelier "Tourner », suggérant des roulades avant sur deux plans inclinés en mousse; un atelier "Se renverser », suggérant des renversements manuels à partir d'un plinth ou d'un plan incliné ; un atelier "Franchir », suggérant des sauts de cheval à l'aide d'un trampoline ; un atelier "Voler », suggérant des sauts à partir d'un mini-trampoline. Les équipements nécessaires aux ateliers étaient alignés sur toute la longueur du gymnase. Dans chaque atelier des fiches étaient à disposition des élèves, elles précisaient les exercices gymniques à travailler et les critères pour les exécuter correctement. Le choix du dispositif par ateliers, fréquent en EPS, constitue, selon nous, une situation privilégiée pour étudier l'activité collective des élèves 
dans la mesure où le dispositif contraint les élèves dispersés en petits groupes à travailler collectivement, en autonomie et en coopération, sans une présence physique continue de l'enseignant à proximité (Gal-Petitfaux et Cizeron, 2005).

Tableau I. Exemple de traitement des matériaux d'observation et d'entretien de l'activité individuelle-sociale de l'enseignant.

\begin{tabular}{|c|c|c|}
\hline $\begin{array}{l}\text { Description des comportements } \\
\text { de l'enseignant en classe }\end{array}$ & Verbatim d'entretien de l'enseignant & $\begin{array}{c}\text { Traitement des matériaux } \\
\text { (intentions, perceptions, } \\
\text { interprétations de l'enseignant) }\end{array}$ \\
\hline $\begin{array}{l}\text { À la } 28^{\text {ème }} \text { minute de la leçon, } \\
\text { l'enseignant s'approche de } \\
\text { l'atelier « Se renverser » pour } \\
\text { aider un élève à renverser son } \\
\text { corps à partir du plinth. En même } \\
\text { temps qu'il tient l'élève par le } \\
\text { bassin pour l'aider à basculer en } \\
\text { avant, il lève la tête et regarde en } \\
\text { direction de l'atelier « Voler » }\end{array}$ & $\begin{array}{l}\text { EG W : « C'est pas facile de voir tout } \\
\text { ce qui se passe dans la classe, alors } \\
\text { il faut arriver à se faire vite une idée } \\
\text { (...) même si je suis en train de } \\
\text { parler à un gamin, souvent je lève } \\
\text { les yeux et je regarde ce qui se } \\
\text { passe ailleurs } \\
\text { CHERCHEUR : et là, tu as repéré } \\
\text { des choses? } \\
\text { EG W : oui, quand j'ai levé le nez, } \\
\text { j'ai vu Soufiane debout sur le plan } \\
\text { incliné alors qu'il fallait } \\
\text { partir accroupi... et un peu plus tard, } \\
\text { je crois, j'ai vu que le triangle en } \\
\text { mousse était couché alors qu'il } \\
\text { devrait être droit pour faire l'ATR } \\
\text { (l'appui tendu renversé) plat dos. } \\
\text { Donc ça, c'est qu'ils ne sont pas } \\
\text { dans ce que j'ai demandé. » }\end{array}$ & $\begin{array}{l}\text { Intentions : } \\
\text { - Contrôler régulièrement l'activité } \\
\text { globale de travail dans la classe } \\
\text { - Se faire une idée rapide de ce que } \\
\text { sont en train de faire les élèves. } \\
\text { Perceptions : } \\
\text { - La position debout de Soufiane sur } \\
\text { le plan incliné n'est pas normale; il } \\
\text { devrait être accroupi. } \\
\text { - Le triangle en mousse est couché } \\
\text { au sol (sans personne dessus) alors } \\
\text { qu'il devrait être droit. } \\
\text { Interprétations: } \\
\text { - Quand un élève montre une } \\
\text { posture sur les agrès non conforme } \\
\text { à ce qui est attendu, c'est qu'il ne fait } \\
\text { pas le travail demandé. } \\
\text { - quand le tapis en mousse n'est pas } \\
\text { à sa place, les élèves ne sont pas } \\
\text { dans le travail demandé. }\end{array}$ \\
\hline
\end{tabular}

Le recueil des données s'est fait par des enregistrements audio-visuels des leçons, afin de rendre compte des actions observables en classe des élèves et du professeur, et de leurs interactions verbales et corporelles. Des entretiens d'autoconfrontation post-leçons (Theureau, 2006) ont permis ensuite de rendre compte de l'activité individuelle-sociale du professeur et des élèves.

Le traitement des matériaux empiriques s'est fait en trois étapes pour comprendre comment se construit l'activité collective de travail dans la classe. Premièrement, la chronologie de chaque activité individuelle-sociale a été reconstituée dans un tableau à deux volets, en mettant en concordance temporelle les retranscriptions des données de communication, la description des actions filmées et les données d'entretien (Tableau 1: colonne 1 et 2). Deuxièmement, dans chaque activité individuelle-sociale, les composantes de la signification (intentions, perceptions, émotions) ont été identifiées (colonne 3) puis regrouper selon une 
démarche inspirée de la "Grounded Theory » (Strauss \& Corbin, 1990). Troisièmement, les activités individuelles-sociales ont été comparées afin d'identifier des formes typiques d'interactions entre l'enseignant et les élèves.

\section{Résultats}

Les résultats montrent que les activités des élèves et de l'enseignant se coordonnent pour produire une activité collective viable conciliable avec les exigences de travail scolaire de l'enseignant. L'analyse fine des intentions, perceptions et interprétations des élèves et de l'enseignant met en évidence que cette coordination repose sur un processus d'ostentation/masquage des actions de chacun. Ce processus apparait de manière saillante et récurrente chez les élèves, comme chez l'enseignant, dans toutes les leçons et tous les ateliers.

3. 1. L'ostentation comme condition d'articulation des activités individuelles de l'enseignant et des élèves

L'activité des élèves en classe apparaît chaotique et désordonnée, marquée par de nombreux décrochages. D'une part, le caractère morcelé de l'activité se manifeste par l'expression d'un spectre très large de comportements produits par les élèves au cours d'une séquence de travail à un atelier gymnique, donnant une impression de désordre, d'une activité discontinue. D'autre part, les verbatim d'entretien permettent de comprendre que cette structuration syncopée de l'activité repose sur un changement continu et brutal des préoccupations des élèves, chacune d'elles ne durant rarement plus de trente secondes.

Par exemple dans l'atelier «Franchir», entre la $38^{\text {ème }}$ et $40^{\text {ème }}$ minutes, Soufiane réalise les sauts demandés, replace le matériel, discute avec ses camarades, shoote dans un tapis, déambule, attend sans rien faire, fait des sauts interdits, lit la fiche, retente le saut demandé, rigole avec ses camarades.

Au-delà de sa grande diversité, l'activité de chaque élève a une organisation stable reflétant une récurrence cyclique d'actions de travail ostentatoires produites intentionnellement en direction de l'enseignant. Bien que discontinues, les actions tournées vers le travail représentent la durée d'occupation la plus importante chez les élèves. Lors des entretiens, les élèves précisent que ces comportements sont liés à deux préoccupations, celle de s'entraîner à faire les exercices afin d'obtenir une note convenable et celle de montrer à l'enseignant qu'ils travaillent pour éviter les problèmes et se faire bien voir par celui-ci.

Extrait d'autoconfrontation (AC) de Fatima : À l'atelier "Tourner», Fatima ne parvient pas à faire les roulades demandées. Elle effectue des roues et explicite : « en fait, je fais ça pour pas que le prof dise que je fais rien, pour lui montrer que je travaille ». 
De plus, les élèves rendent visibles leurs comportements qui sont conformes au travail demandé afin que l'enseignant, par la suite, tolère quelques moments de détente. Dans leur atelier, les élèves cherchent toujours à repérer la position de l'enseignant. Lorsque ce dernier se situe à proximité, ils saisissent l'occasion pour attirer son attention : "Monsieur, monsieur, regardez ! ». Dans ce cas là, leur intention vise d'une part à montrer à l'enseignant qu'ils sont en train de travailler, et d'autre part obtenir une validation de sa part sur ce qu'ils vont ou viennent de réaliser.

AC de MEGDA : « Je vais faire ma roulade et je vois que le prof, il est à côté, j'en profite pour lui demander si c'est bien (ce que je fais).

CHERCHEUR : Et là, tu essaies de le faire bien, l'exercice ?

MEGDA : Oui, faut lui montrer que je m'applique, que je fais attention à tout ce qu'il a dit...»

Pour les élèves, c'est important de montrer à l'enseignant qu'ils travaillent, car cela évite les réprimandes trop importantes lorsque qu'ils se font prendre à ne pas faire ce qui est demandé.

AC de Megda : « ici, je m'amuse un peu, le prof il peut rien me dire, il a vu que je travaillais ».

L'activité de l'enseignant reflète elle aussi une structuration typique. II exprime de manière ostentatoire son intérêt pour le travail effectué. Malgré une agitation constante dans la classe, la majorité de ses interventions verbales portent sur des conseils liés aux exercices demandés plutôt que sur des réprimandes. En entretien, l'enseignant exprime être attentifs aux transgressions des élèves, pour autant il se force à intervenir auprès des élèves qui manifestent un engagement dans le travail demandé.

AC de EG W. : « j'interviens sur Ines car elle essaie de faire l'exercice,

Chercheur : tu interviens sur Ines et pas sur Megda?

EG W. : Megda là elle est "out", elle est pas dans la tâche, ça m'énerve mais je veux me concentrer sur les élèves qui travaillent sinon je passe mon temps à les recadrer et finalement je n'enseigne rien. »

En intervenant ostensiblement sur le travail fourni par les élèves, l'intention de l'enseignant est de concentrer leur attention sur le travail et de ne pas attirer leur attention sur les déviances en les réprimandant. II cherche ainsi à engager les élèves dans une dynamique positive d'apprentissage et de progrès, plutôt que dans une spirale négative de répression.

AC de EG W : « J'interviens sur les contenus et pas sur l'organisationnel parce que c'est l'essentiel. Je me force à revenir sur les contenus parce que les élèves ça les intéresse. Ils disent "ah ben oui, je savais pas le faire et j'ai écouté le professeur et maintenant ça va mieux". Donc quelque part, ils progressent et derrière, moi, j'ai moins de problèmes de comportement parce qu'ils sont intéressés. (...) C'est dans 
l'intérêt général, s'ils ne progressent pas, je sens très vite que ça va devenir très pénible après, dans la classe. "

De plus, l'enseignant rend délibérément visible le travail à faire à travers de la disposition matérielle. Pour engager les différents groupes dans le travail à réaliser, il circule d'un atelier à un autre et vérifie de façon prioritaire que le matériel reste au bon emplacement et dans une position conforme à celle exigée pour effectuer les exercices gymniques. Dans le cas où le matériel est mal placé, il attire publiquement l'attention des élèves du groupe concerné et indiquant explicitement comment installer le matériel ; cette forme d'intervention publique lui permet d'attirer l'attention des élèves du groupe en question, et des groupes voisins, sur les apprentissages à réaliser en lien avec les objets.

EG W. : « Ce tapis il n'a rien à faire là, alors je l'enlève et le plan incliné (je le repositionne), ils jouent plus avec qu'ils ne travaillent, je vais les recentrer...

CHERCHEUR : C'est frappant, dès que tu arrives (dans l'atelier), tu mets en place le matériel... ça a une importance particulière pour toi l'organisation matérielle?

EG W. : Ça donne un cadre... ça va cadrer leur comportement, le matériel va leur permettre de savoir ce qu'ils doivent faire... Là par rapport au tapis rouge, ils savent qu'ils doivent passer les bras derrière pour aller chercher le plus loin possible. »

De même, l'enseignant centre les élèves sur le travail par un usage ostentatoire des fiches dans chaque atelier. Lorsqu'il intervient auprès des élèves l'enseignant fait souvent référence aux fiches, il la lit publiquement pour mettre en évidence les différents exercices à faire et leurs critères de réalisation. Son intention est ici de rendre visible aux élèves que la fiche est un substitut de l'enseignant pour s'informer du travail à faire. Ce qui lui permet de concentrer sa disponibilité pour aider les élèves à apprendre les habiletés gymniques.

EG W. : « Là Mohamed, il n'a pas lu la fiche, je n'ai pas de temps à perdre avec lui, je lui dis "retourne voir la fiche, tout est écrit"... là la priorité c'est Maureen, elle a peur de faire la roulade, si je suis pas derrière elle, elle va rien faire ! (...) Tu as vu, Mohamed est retourné au travail. Ici il n'a pas eu besoin de moi, il s'est servi de la fiche pour savoir ce qui lui restait à faire. »

Enfin, la forme des interventions de l'enseignant est caractéristique de son activité de mise en visibilité publique du travail à effectuer. Lorsqu'il corrige, aide, encourage un élève qui travaille, l'enseignant le rend visible pour intéresser les élèves alentour, par ses gestes, son ton, son regard et son adressage.

Par exemple, il explicite ses actions dans l'atelier "Se renverser » lorsqu'il corrige Anthony. Son geste doigt tendu pointe le renversement exemplaire de l'élève afin de montrer aux autres ce qu'il attend d'eux. De plus, son ton est fort pour être audible par les élèves éloignés de lui. Son regard est dirigé vers le reste du groupe, et il utilise le " on » et le "vous " pour attirer l'attention collective : "Super Anthony. Vous 
voyez, c'est super ce renversement. On regarde la position des mains et il est bien droit comme un bâton ».

Cet épisode de classe montre qu'en plus de se focaliser sur les savoirs à acquérir, l'enseignant théâtralise ses interventions afin de les rendre publiques, cela lui permet de raccrocher (ou maintenir) plus efficacement au travail les élèves qui s'en détournent ponctuellement. II recourt à des pratiques d'ostentation : il use alors simultanément de la gestualité et de l'oralité, pour récupérer l'attention des élèves non concernés et concentrer l'attention collective sur le travail. Par exemple dans l'extrait précédent, il pointe fermement et ostensiblement le doigt tendu dans la direction de l'élève pour indiquer, et montrer à tous, le point positif à considérer; par une dissociation du geste bras tendu (pointant un aspect qualitatif de la prestation d'un élève) et de son regard (dirigé vers le reste du groupe), il rend public pour tous les savoirs à acquérir; par l'usage d'un ton fort, audible par des élèves relativement éloigné de lui, il attire l'attention collective sur ses propos ; enfin, par l'emploi récurrent du " on " impersonnel, du "vous " ou de "tout le monde », il commet un acte directif dirigé vers le collectif, lui permettant de ré-évoquer les acquisitions attendues pour tous. Les actions d'ostentation, dirigés sur ce qu'il y a à apprendre, servent de ciment à l'instauration d'une activité collective studieuse et une communauté d'apprentissage dans chaque atelier, et par extension, dans la classe. L'enseignant concentre en permanence l'attention collective des élèves sur ce qu'il y a à apprendre, grâce à : une mise en visibilité spatiale, corporelle et orale, de ce que les élèves doivent savoir; une association systématique entre une consigne d'apprentissage et une action corporelle particulière (geste, accentuation tonale, position spatiale, direction du regard) pour impliquer les élèves ; une instruction collective, conduite simultanément pendant des interventions individualisées. De ce mode typique d'adressage mixte (individuel-collectif et oral-gestuel), émerge un format particulier d'interaction, au service d'une activité collective dans la classe.

3. 2. Le masquage comme condition d'articulation des activités individuelles de l'enseignant et des élèves

L'activité collective dans la classe ne repose pas seulement sur l'ostentation de leurs actions par l'enseignant ou les élèves, elle est aussi conjointement structurée par un processus de masquage. Les pratiques de masquage permettent de concilier les activités individuelles contradictoires des élèves et de l'enseignant. Elles sont présentes $1 /$ chez les élèves qui camouflent leurs actions déviantes aux yeux de l'enseignant et $2 /$ chez l'enseignant qui masque son contrôle de la classe face aux élèves. 
Du point de vue des élèves, même si leur engagement au travail est dominant, leur activité est marquée de manière récurrente par de nombreux décrochages. Les résultats montrent que ces décrochages ont un caractère systématique, malgré leur grande variété. L'activité des élèves repose sur deux formes typiques de décrochage que sont l'errance et le jeu. D'une part, les actions des élèves manifestent une « errance » à la fois a) spatiale, quand les élèves déambulent, et b) psychologique, quand ils explicitent « je ne sais pas quoi faire » ou qu'ils avouent éprouver un sentiment d'ennui. Cette errance se traduit par des comportements apathiques d'attente et/ou d'observation de ce qui se passe autour d'eux. D'autre part, les élèves décrochent du travail scolaire pour des actions ludiques marquées par des jeux entre pairs. Leur intention est de « s'amuser à se taquiner, se faire des coups en douce » et se mettre " en spectacle devant ses amis ».

Pour autant, en restant camouflés, ces décrochages ne mettent pas en péril l'activité collective de travail. Autrement dit, pour ne pas se faire repérer, les déviances des élèves sont a) furtives, b) masquées aux yeux de l'enseignant, c) dans l'espace de travail prescrit, d) intégrées à la tâche demandée. Ces quatre caractéristiques typiques des pratiques de masquage des élèves rendent les actions déviantes minoritaires par rapport à celles tournées vers le travail. En ce sens, c'est parce que les élèves cherchent à masquer leurs déviances que celles-ci demeurent furtives et moins fréquentes, ce qui lui permet de s'articuler avec l'activité de l'enseignant.

Tout d'abord, les élèves masquent leurs actions déviantes en leur donnant un caractère furtif. Afin de diminuer les chances de se faire repérer par l'enseignant, ils ne restent pas longtemps engagés dans une même action. Ils changent sans cesse leurs préoccupations en alternant actions de travail et actions de jeu et d'errance.

Ensuite, afin ne pas être vu par l'enseignant, les élèves contrôlent où il est positionné avant d'effectuer une déviance. Ils repèrent en particulier dans quel atelier il se situe et quelle est la position de ses épaules. Dans l'exemple suivant, Mohamed a repéré le déplacement de l'enseignant qui va à l'autre bout de la salle en leur tournant le dos. Cette position fait signe pour lui et ouvre l'opportunité de s'amuser avec son camarade.

AC de Mohamed qui commente sa course d'élan à l'atelier « Franchir » : " Ouais, on fait la course avec Gary, on a vu que le prof il a le dos tourné, alors on en profite pour rigoler un peu ».

De plus, les opportunités de ces actions déviantes sont indexées à des espaces bien particuliers. La file d'attente pour prendre son tour de passage à l'atelier est notamment un espace favorable à l'émergence de comportements déviants. La position statique de l'élève, 
et sa proximité avec les autres élèves en attente, génèrent des contacts physiques et des interactions verbales entre les élèves. Ils en profitent aussi pour s'amuser avec le matériel à proximité. Par exemple, le plot qui matérialise l'endroit où doit se tenir le premier élève de la file d'attente est souvent saisi pour mimer un porte voix ou un chapeau posé sur la tête. Ou encore, un élève occupe le tremplin en se tenant immobile dessus, dans le but d'empêcher son camarade, en tête de file, de passer.

CHERCHEUR : « Et là dans la file d'attente, tu pousses Damien ?... qu'est que tu fais ?

AC de ROMAIN : (rire) Oui, je l'ai poussé dans le dos... c'est un jeu entre nous, on s'amuse à se faire des coups en douce.

CHERCHEUR : Et vous n'avez pas peur que le prof vous gronde?

ROMAIN : Le prof, il peut rien dire, on fait rien de mal, je lui fais juste une petite poussette dans le dos... en plus, je suis caché derrière Ali, le prof, il peut pas nous voir. »

Enfin, ces décrochages sont difficilement repérables par l'enseignant car ils sont intégrés à la tâche demandée. Les élèves produisent leurs déviances dans l'espace de travail de l'atelier et avec le matériel prévu pour l'exécution de la tâche, ce qui contribue à les masquer aux yeux de l'enseignant.

Par exemple, dans l'atelier « Voler », les élèves tentent fréquemment des saltos alors que l'enseignant a demandé des «sauts droits ». L'observation fine montre que les actions déviantes de sauts (les saltos) ne durent qu'une fraction de seconde alors que le reste des actions (la réception du saut, le retour dans la file d'attente, l'attente pour passer, la course d'élan) se déroulent conformément aux consignes de l'enseignant et peuvent occuper une durée proche de 1 minute... Ce résultat invite à interpréter que si l'enseignant ne jette un coup d'œil que d'une seconde, il n'a qu'une chance sur soixante de repérer le saut déviant imbriqué dans le cours des autres actions scolaires conformes.

Les quatre caractéristiques typiques du masquage des actions déviantes (furtivité, cachée aux yeux de l'enseignant, localisée dans l'espace de travail prescrit, intégrée à la tâche demandée) rendent les déviances à la fois minoritaires et difficiles à percevoir pour l'enseignant. Cette pratique de masquage donne une forme typique à l'activité individuellesociale des élèves qui est compatible avec les attentes de l'enseignant.

L'activité de l'enseignant s'articule avec celle des élèves car a/ il tolère certaines déviances mineures et b/ il camoufle volontairement son contrôle de la classe. La tolérance de l'enseignant à certaines de ces activités favorise l'engagement collectif des élèves dans le travail et devient paradoxalement, dans certaines limites, une condition de leurs apprentissages. Pour lui, cette marge de liberté d'action, même déviante, laissée aux élèves 
est une condition pour que par la suite, ceux-ci s'engagent dans l'exercice prescrit et maintiennent leur adhésion sans se " mettre à l'écart ".

AC de EG W: " Ils ont besoin de ça. S'il n'y avait pas ces jeux... ces temps d'amusement et que c'était roulade, roulade, ça ne les intéresserait pas et les élèves se mettraient de côté. Donc, plutôt que de les voir ne rien faire, je préfère qu'il y ait une partie travail et une partie jeu. Si je me mettais à coté d'eux et que je disais "aucune activité parallèle, aucun travers", ils diraient "oh, si c'est ça, je fais rien, je me mets sur le côté". ». "C'est la soupape de sécurité. ». "À la limite c'est presque mieux ça, plutôt qu'ils ne fassent rien, au moins ils bougent, au moins ils sont actifs, donc tu te dis que si pendant 30 secondes ils soufflent un peu, après ils vont se remettre au boulot. »

Cet extrait montre que ces activités parallèles ne remettent pas en cause l'engagement de la classe au travail, au contraire, elles l'entretiennent en lui permettant de se poursuivre. Ces amusements ont lieu entre deux temps de travail et, pour l'enseignant, ils vont souvent s'estomper d'eux-mêmes par lassitude des élèves. Cette tolérance de l'enseignant caractérise un autre aspect typique de la forme des interactions qu'il construit dans la classe, et elle contribue à rendre viable une activité collective de travail chez les élèves. C'est parce que les élèves peuvent justement décrocher furtivement du travail prescrit, dans un seuillimite, que cette activité collective devient pérenne.

De même, pour maintenir une activité collective de travail dans la classe, il camoufle son contrôle de la classe. II masque volontairement sa surveillance des comportements des élèves pour éviter de rentrer en conflit avec eux et de provoquer des décrochages durables. II précise que ce type d'élèves difficiles « ne supporte pas d'être fliqué. Si je reste toujours derrière eux, soit ils arrêtent, soit ils entrent en conflit ». Ainsi, il contrôle l'ambiance de travail de la classe de manière masquée, pour cela il s'appuie sur a) la disposition des ateliers et b) des interactions typiques.

Tout d'abord, il masque sa surveillance par une disposition particulière des ateliers. Lors de l'installation du matériel de gymnastique, l'enseignant a contraint les élèves à disposer les ateliers en les espaçant nettement les uns à côté des autres contre le mur. Par un écartement suffisant entre eux, il empêche que les élèves d'un atelier ne croisent ceux des ateliers voisins et chahutent, sous peine d'être repérés immédiatement par l'enseignant. II les circonscrit ainsi dans un espace bien délimité, afin de les concentrer sur le travail à réaliser dans leur atelier.

EG W. : « Je sépare bien les ateliers... comme ça c'est rangé, les élèves sont bien en place (...) Cette espèce de cloisonnement par atelier, ça permet déjà de voir, de relativement bien situer les gamins, quand il y en a un qui n'est pas sa place je vois tout de suite. » 
Ainsi cette disposition rend saillante la localisation spatiale des élèves. Sans une surveillance accrue, par un simple coup d'œil sur la disposition des élèves par rapport aux ateliers, l'enseignant contrôle la circulation des élèves entre les ateliers. De plus, chaque atelier a son organisation propre, les élèves ont un chemin de circulation bien précis. Par exemple dans l'atelier "Franchir ", ils doivent enchaîner une course d'élan limitée à cinq mètres, un saut et un retour dans la zone de départ. Cette rotation régulière des élèves dans les ateliers est, pour l'enseignant, significative d'un bon fonctionnement. En revanche, des circulations en dehors de ces espaces, ou inversement l'immobilité d'un groupe, sont des éléments qu'il perçoit rapidement comme étant non conformes. En un coup d'œil sur l'ensemble de la classe, l'enseignant se fait une idée de l'ambiance de travail. En observant l'occupation de l'espace et la mobilité des élèves, il évalue comment la classe travaille.

Pour l'enseignant, quand la classe travaille, il y a des élèves immobiles dans les files d'attente parallèles, et des élèves en mouvement qui se dirigent perpendiculairement par rapport au mur. Cette forme spatiotemporelle est, pour lui, un repère de l'activité collective de travail des élèves.

La délimitation des espaces de travail conduit les élèves à occuper des zones précises, et conditionne leur engagement dans le travail à la fois au niveau de chaque atelier et de l'espace de la classe. Ainsi, il se construit la possibilité d'une surveillance rapide et camouflée aux yeux des élèves. L'articulation entre son activité de surveillance dissimulée et l'activité des élèves est rendue saillante par l'intermédiaire de l'organisation spatiale. Ainsi, cette dernière participe à la construction de l'activité collective de travail dans la classe.

Ensuite, son activité de contrôle du travail de la classe est masquée car elle est imbriquée dans ses interventions d'instruction auprès des élèves. Pendant qu'il aide un élève à apprendre un exercice gymnique au sein d'un atelier, il s'oriente toujours de façon à pouvoir jeter un coup d'œil discret sur les autres ateliers et vérifier que les autres travaillent.

AC de EG W. : "C'est pas facile de voir tout ce qui se passe dans la classe, alors il faut arriver à se faire vite une idée (...) même si je suis en train de parler à un gamin, souvent je lève les yeux et je regarde ce qui se passe ailleurs

CHERCHEUR : et là, tu as repéré des choses?

EG W. : oui, quand j'ai levé le nez, j'ai vu Soufiane debout sur le plan incliné alors qu'il fallait partir accroupi...»

De plus, le contrôle discret de l'enseignant s'effectue à des moments opportuns. Dans l'interaction, lorsqu'il est en train d'aider un élève, il a deux instants privilégiés pour contrôler ce que font les autres élèves, 1) lorsque l'élève s'ajuste pour passer et 2) juste après son passage, pendant qu'il donne des retours sur la réalisation. C'est à ces moments précis que 
l'enseignant jette des coups d'œil rapide sur le reste de la classe, ou sur un groupe qui a attiré son attention.

Par l'exemple l'enseignant contrôle le groupe de l'atelier « Voler» tout en intervenant dans l'atelier " Se renverser». Lorsque Inès s'allonge sur le ventre pour faire son renversement, l'enseignant jette un premier coup d'œil sur l'autre atelier, Olivier est sur le tapis de réception, il vient de se réceptionner de son saut. L'enseignant continue de surveiller le groupe car il n'a pas pu juger de la nature et de la qualité du saut. Juste après le renversement d'Inès, l'enseignant dit « c'est mieux, tiens-toi droite comme un bâton » tout en regardant l'atelier voler.

Même si l'enseignant ne regarde que furtivement les élèves des autres ateliers, il garde en continu un contrôle auditif du reste de la classe. Un bruit qui sort de l'ordinaire, un cri strident par exemple sont directement perçus comme significatifs pour lui d'une ambiance de travail qui se détériore.

AC de EG W. : « Oui là j'étais sur les contenus du placement de main, bassin,... (...) et j'entends un bruit aigu. Hop, je regarde (ce qui se passe) (...) c'est un cri qui n'a rien à faire dans un atelier de toutes façons, c'est un cri qui veut dire amusement ou conflit entre les élèves.

CHERCHEUR : Alors, malgré le fait que tu interviennes dans un atelier de manière spécifique auprès des élèves sur la tâche à réaliser et sur l'investissement de la fille qui n'était pas prête à le faire, tu es pris visuellement mais tu restes à l'écoute de l'ambiance...

EG W. : De ce qui se passe, oui tu entends. "

Quand l'enseignant sent que la situation de classe est relativement contrôlable, il évite d'intervenir directement sur les écarts de conduite des élèves. II veille volontairement à masquer son intention d'avertissement ou de sanction. II opère alors de façon détournée : soit il leur laisse croire qu'il ne les voit pas, tout en contrôlant que ces déviances légères s'autorégulent d'elles-mêmes; soit il contraint les déplacements dans l'espace en leur signifiant qu'ils ne sont pas "à leur place", pour ne pas interrompre sans cesse la dynamique du cours. II utilise des gestes professionnels routiniers en pointant fermement et ostensiblement le doigt tendu dans la direction où l'élève doit aller; ou bien, en jetant vers lui un regard appuyé et perçant (il tend son bras, paume de main ouverte et face dirigée vers l'élève, pour lui signaler qu'il faut stopper immédiatement) ; ou en se déplaçant vers l'élève en hochant la tête pour signifier un "Non ». Tout en réalisant ces gestes, l'enseignant continue ainsi d'intervenir sur les savoirs gymniques. Alors même qu'il rend visible à un élève qu'il vient de repérer son écart de conduite (sans pour autant rendre public sa désapprobation), il reste focalisé simultanément sur un autre élève qu'il guide pour apprendre l'habileté gymnique prescrite. Ainsi à l'aide de ce langage corporel, il peut intervenir à la fois de façon discrète sur le cadrage spatiale et ostensiblement sur les apprentissages gymniques. 
EG W. : « J'essaie de le regarder, le fixer puis ça peut suffire pour le remettre en place. »

Néanmoins, lorsque l'activité collective de travail de la classe est mise en péril, l'enseignant rend public son contrôle. II juge in situ de la viabilité de l'engagement au travail des élèves selon trois seuils limite. II interviendra directement dans le cas a) où les déviances des élèves durent dans le temps, b) où il y a un problème de sécurité, c) où les interactions entre élèves deviennent délétères et nuisible au travail dans la classe. Dans ces situations, il tente de rétablir rapidement l'activité collective de travail par sa circulation et ses réprimandes ponctuelles. Lorsqu'il sent que l'ambiance de travail de la classe se dégrade, il circule rapidement dans les ateliers avec l'intention de se faire voir par les élèves et il utilise un mode de réprimandes publiques afin de montrer qu'il y a des limites strictes qu'il ne faut pas dépasser.

Nous venons de voir, qu'au delà de ces trois seuils limites, l'enseignant évite de s'opposer directement aux écarts de conduite des élèves. Plutôt que d'intervenir directement et publiquement par des rappels à l'ordre pour contrer à chaque instant ces écarts, il les laisse s'exprimer dans une limite contrôlée pour que ces déviances légères s'autorégulent d'ellesmêmes. Tant que les élèves ne transgressent pas de façon exagérée et durable les règles du travail, il intervient alors de façon détournée en masquant sa surveillance. Cette activité de surveillance, diffuse et secrète, lui permet d'éviter des conflits avec les élèves qui se sentiraient persécutés. Elle participe aussi à la construction d'une activité collective de travail dans la classe.

\section{Conclusion}

Les résultats ont montré que malgré des activités individuelles contrariées typiques des classes en Réseau Ambition Réussite, vectrices de tensions et expliquant notamment la pénibilité du travail enseignant, une activité collective de travail peut s'installer et perdurer. La dimension d'ostentation et de masquage de l'activité apparaît comme le fondement de l'activité collective ; elle permet d'articuler les activités concurrentes de l'enseignant et des élèves. Les activités individuelles parviennent à se coordonner parce que les élèves montrent à l'enseignant qu'ils fournissent du travail, tout en parvenant à lui masquer leurs déviances ; de son côté, l'enseignant dévoile aux élèves qu'il s'intéresse au travail qu'ils fournissent, tout en cachant le contrôle de leurs déviances. L'activité collective de travail dans une classe de RAR a typiquement une dynamique d'activités contrariées, dans laquelle les acteurs ajustent 
en permanence leurs activités individuelles pour éviter qu'elles ne deviennent contradictoires. La coordination des activités individuelles repose ainsi sur des ajustements locaux (Vors \& Gal-Petitfaux, 2008b) et non sur une «we-intention » (Searle, 1990). En d'autres termes, l'activité collective n'est pas tenue par une intentionnalité collective commune à tous les acteurs de la classe, nous avons vu que bien souvent l'intention des élèves diverge de celle de l'enseignant. Ces divergences participent aussi de la viabilité d'une situation collective. Tous les groupes sont constitués de synergies et d'antagonismes nécessaires à leur existence durable, ces tensions permettant de concilier les satisfactions personnelles des acteurs et les exigences du travail collectif (Élias, 1991). Ces analyses convergent vers l'idée d'îlots de compréhension locale (Bourbousson, Poizat, Saury \& Sève, 2008) plutôt qu'une intentionnalité commune à toute la classe. De même, comme l'évoque Lave et Wenger (1991), l'activité d'apprentissage correspond plus à un engagement congruent qu'à un partage de représentation identique entre l'enseignant et l'apprenant. C'est le processus d'ostentation/masquage qui rend congruent l'engagement des participants. L'apprentissage des élèves est indexé à leur forme de participation dans l'environnement social et matériel de la classe ; ici, il est structuré par des actions de travail de la tâche codéterminées par des actions déviantes (Guérin, Pasco \& Riff, 2008).

La forme typique des coordinations liées au couplage ostentation/masquage repose sur une configuration d'activité (Durand, Saury \& Sève, 2006) propice au travail qui articule les actions de l'enseignant avec les actions des élèves, dans le contexte particulier du travail par ateliers. La configuration d'activité correspond à un degré d'intelligibilité supérieur de la situation, qui dépasse la compréhension de chacun des acteurs. C'est une totalité englobant les activités individuelles qui sont mutuellement codéterminées et indexées au contexte spatial (Veyrunes, Gal-Petitfaux \& Durand, 2007).

Cette étude ouvre trois pistes de réflexion sur la formation des enseignants. D'une part, elle montre la nécessité de regarder les situations d'enseignement scolaires comme interpénétrées avec le réseau du contexte spatial de la classe et des objets qui y sont disposés. D'autre part, cette étude a mis en évidence un geste professionnel relatif à l'autorité des enseignants expérimentés. II se caractérise par la compétence à masquer le contrôle des déviances dans la classe tout en fondant l'autorité auprès des élèves sur les connaissances à apprendre. Ce savoir pratique est particulièrement adapté dans ces milieux d'éducation prioritaire où l'autorité autoritariste est souvent vectrice de conflit (Robbes, 2008). Enfin, elles attirent l'attention sur une compétence fondamentale nécessaire à l'exercice du métier 
d'enseignant, notamment dans les établissements difficiles : celle de savoir agencer un espace de travail, de savoir s'y déplacer et d'en exploiter habilement les propriétés matérielles, pour installer et maintenir tout au long d'une leçon des conditions favorables au travail collectif dans la classe (Gal-Petitfaux \& Vors, sous presse).

\section{Bibliographie}

Amigues, R., \& Zerbato-Poulou, M.-T. (2000). Comment l'enfant devient élève : les apprentissages à l'école maternelle. Paris : Retz.

Arripe-Longueville, F. (d'), Fleurance, P., \& Winnykamen, F. (1995). Effects of the degree of competence symmetry asymmetry in the acquisition of a motor skill in a dyad. Journal of Human Movement Studies, 28, 255-273.

Baluteau, F. (2003). École et changement: Une sociologie constructiviste du changement. Paris : L'Harmattan.

Barbier, J.M., \& Durand, M. (2003). L'activité : un objet intégrateur pour les sciences sociales ? Recherche et Formation, 42, 99-117.

Bourbousson, J., Poizat, G., Saury, J. \& Sève, C. (2008). Caractérisation des modes de coordination interpersonnelle au sein d'une équipe de basket-ball. @ctivités, 5 (2), 219.

Chauveau, G. (2001). La réussite scolaire dans les ZEP. Éducation et Formations, 61, 147151.

Coulon, A. (1988). Ethnométhodologie et enseignement. Revue Française de Pédagogie, 82, 65-101.

Doyle, W. (1977). Learning the classroom environment: An ecological analysis. Journal of Teacher Education, 28, 51-55.

Durand, M., Saury, J., \& Sève, C. (2006). Apprentissage et configuration d'activité : une dynamique ouverte des rapports sujets-environnements. In J.M. Barbier \& M. Durand (Eds.), Sujets, activités, environnements : approches transverses (pp. 61-84). Paris : PUF.

Élias, N. (1991). La société des individus. Paris : Fayard, 1991. 
Filippi, G. (1994). La construction collective de la régulation du trafic du RER. Étude ergonomique dans une perspective de conception de situation d'aide à la coopération. Thèse de doctorat en ergonomie, Paris: Université Paris Nord.

Gal-Petitfaux, N., \& Cizeron, M. (2005). Le travail par ateliers en Éducation Physique : activité de l'enseignant et activité des élèves. Actes du Colloque International Recherche et Formation «Former des enseignants - professionnels, savoirs et compétences ». Nantes : IUFM.

Gal-Petitfaux, N., \& Vors, O. (2008). Socialiser et Transmettre des savoirs en classe d'Education physique: une synergie possible au prix d'une autorité pédagogique conciliante. Éducation et Francophonie, XXXVI : 2, 118-139.

Gal-Petitfaux, N., \& Vors, O. (sous presse). Le rôle des objets dans l'articulation d'activités publiques et masquées participant à la viabilité d'une situation d'enseignement : une étude en gymnastique scolaire. In D. Adé, \& I. de Saint-Georges (Eds.), Les objets dans la formation et l'apprentissage : usages, rôles et significations dans des contextes variés. Paris : Octarès.

Glasman, D. (2003). Quelques acquis d'un programme de recherches sur la déscolarisation. Revue Ville-Ecole-Intégration, 132, 8-18.

Goffman, E. (1991). Les Cadres de l'Expérience. Paris : Éditions de Minuit.

Guérin, J., Pasco, D., \& Riff, J. (2008). Activités dissimulée et publique d'un élève décrocheur en mathématiques. Revue des Sciences de l'éducation pour l'ère nouvelle, 41(1), 6382.

Hastie, P. A., \& Siedentop, D. (2006). The classroom ecology paradigm. In D. Kirk, M. O'Sullivan, \& D. Macdonald (Eds.). The Handbook of Physical Education, (pp. 214225). London: Sage.

Hutchins, E.A. (1995). Cognition in the wild. Cambridge: The MIT Press.

Joseph, I. (1998). Erving Goffman et la microsociologie. PUF : Paris.

Kherroubi, M., \& Rochex J.Y. (2004). Note de synthèse. La recherche en éducation et les ZEP en France. (2) Apprentissages et exercice professionnel en ZEP : résultats, analyses, interprétations. Revue Française de Pédagogie, 146, 115-190.

Lave, J., \& Wenger, E. (1991). Situated Learning: legitimate peripheral participation. Cambridge: Cambridge University press.

Lahire, B. (2004). La culture des individus. Dissonances culturelles et distinction de soi. Paris : La découverte. 
Leplat, J. (1994). Collective activity in work some lines of research. Le Travail Humain, 57, 209-226.

Marchive, A. (2003). Ethnographie d'une rentrée en classe de cours préparatoire: comment s'instaurent les règles de la vie scolaire ? Revue Française de Pédagogie, 142, 21 32.

Millet, M., \& Thin, D. (2005). Ruptures scolaires : L'école à l'épreuve de la question sociale. Paris: PUF.

Monfroy, B. (2002). La définition des élèves en difficulté en ZEP : le discours des enseignants de l'école primaire. Revue française de pédagogie, 140, 33-40.

Mottier Lopez, L. (2006). Microculture de classe et interactions collectives dans la résolution de problèmes mathématiques. In P. Dessus, \& E. Gentaz (Eds.), Apprentissages et enseignement, sciences cognitives et éducation (pp. 201-218). Paris: Dunod.

Newell, A., \& Simon, H. A. (1972). Human problem-solving. Englewood Cliffs, N.J : Prentice Hall.

Robbes, B. (2008). La relation d'autorité éducative: des savoirs d'actions efficaces et contextualisés. Communication à la IV conférence mondiale sur la violence à l'école, Lisbonne.

Salembier, P., \& Zouinar, M. (2004). Intelligibilité mutuelle et contexte partagé. Inspirations conceptuelles et réductions technologiques. @ctivités, 2, 64-85.

Sartre, J.-P. (1960). Critique de la raison dialectique. Paris : Gallimard.

Saury, J. (2008). La coopération dans les situations d'intervention, de performance et d'apprentissage en contexte sportif. HDR STAPS, non publiée. Nantes : Université de Nantes.

Searle, J. R. (1990). Collective intentions and actions. In P. R. Cohen, J. Morgan, \& M. E. Pollak (Eds). Intentions in Communication, (pp. 401-415). Cambridge: MIT Press.

Sperber, D., \& Wilson, D. (1986). Relevance. Communication and cognition. Cambridge: Harvard University Press.

Strauss, A. \& Corbin, J. (1990). Basics of qualitative research: Grounded theory procedures and techniques. London: Sage.

Theureau, J. (2006). Le cours d'action. Méthode développée. Toulouse: Octarès.

Theureau, J. (2009). Le cours d'action. Méthode réfléchie. Toulouse: Octarès.

Veyrunes, P., Gal-Petitfaux, N., \& Durand, M. (2007). La lecture orale au cycle 2: configuration et viabilité de l'activité collective dans la classe. Repères, 36, 59-76. 
Vors, O., \& Gal-Petitfaux, N. (2008a). La socialisation scolaire des élèves en 'Réseau ambition réussite' : une construction articulée avec des formes de sociabilité primaire conflictuelles. Actes du colloque "Compétences et socialisation», organisé par le CERFEE de I'Université Montpellier 3 et LIRDEF de l'IUFM de l'académie de Montpellier, Montpellier, 7, 8 septembre 2007.

Vors, O., \& Gal-Petitfaux, N. (2008b). Mettre une classe au travail en Réseau Ambition Réussite : des formes typiques d'interaction enseignant-élèves lors de leçons d'EPS. Travail et formation en éducation, 2. [En ligne], mis en ligne le 18 décembre 2008. URL : http://tfe.revues.org/index724.html. Consulté le 28 avril 2009. 